\title{
DEVELOPMENT OF ALGORITHM MODEL FOR EXHAUST GASES SYSTEM OF DIESEL ENGINE WITH ELECTRONIC CONTROL DiAgNOSTICS
}

\author{
Miroslav Grubisic, Boris Crnokic
}

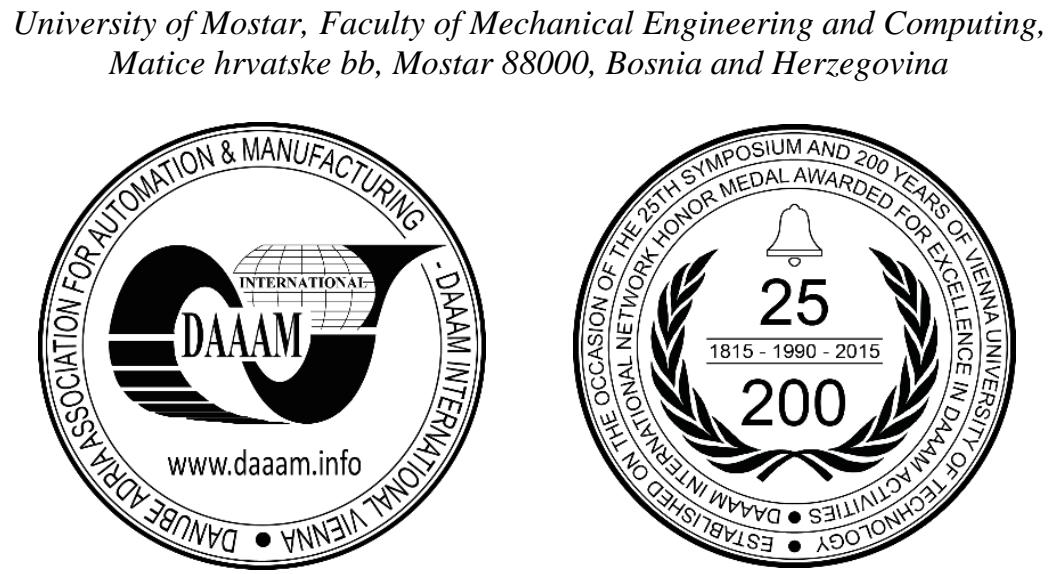

This Publication has to be referred as: Grubisic, M[iroslav] \& Crnokic, B[oris] (2016). Development of Algorithm Model for Exhaust Gases System of Diesel Engine with Electronic Control Diagnostics, Proceedings of the 27th DAAAM International Symposium, pp.0768-0774, B. Katalinic (Ed.), Published by DAAAM International, ISBN 978-3-90273408-2, ISSN 1726-9679, Vienna, Austria

DOI: $10.2507 / 27$ th.daaam.proceedings.111

\begin{abstract}
This paper discusses the problem of increased emissions of harmful exhaust gases of the Diesel engine in situations where the engine control unit (ECU) has not memorized a single error that indicates a malfunction in the control system of the engine. In order to detect hidden problems in the Diesel engine performance, which directly affect the increase in its emissions, this paper has developed an optimal algorithmic model for system diagnostics of Diesel engines with electronic control exhaust gases after-treatment. Functionality check of developed aphorism model was conducted experimentally on Diesel motors with electronic control and built-in exhaust gas recirculation into the intake engine manifold (EGR), oxidation catalyst, diesel particulate filter (DPF) and selective catalytic reduction (SCR) system.
\end{abstract}

Keywords: Diesel engine; exhaust gases; diagnostic model; exhaust gas recirculation; EGR; diesel particulate filter; DPF; selective catalytic reduction; SCR

\section{Introduction}

Diesel engine is undoubtedly one of the most important inventions of the nineteenth century and the fact is that a large part of the world system of transportation of people and goods is based on it. On the other hand, in the last years of the twentieth century was definitely, by means of analysis and monitoring, found that motor vehicles driven by internal combustion engines, besides the industry, are the biggest air pollutants due to increased emissions of harmful combustion products and greenhouse gas emissions. This emission has a direct impact on human health, which is particularly acute in urban city environments. Diesel engine has its own harmful emissions, such as carbon monoxide (CO), unburned hydrocarbons (HC), nitrogen oxides (NOx) and particulates of soot (PM). As a product of combustion in the Diesel engine, there is the emission of carbon dioxide $\left(\mathrm{CO}_{2}\right)$ called greenhouse gas that leads to a warming of the earth due to the greenhouse effect. 
In order to protect the environment, the European Union has, to all the manufacturers of vehicles with Diesel engines, imposed requirements (Euro norms) in terms of emissions. These legal requirements strictly determine how much harmful combustion products the Diesel engine exhaust gases can contain in various conditions of use [1]. Having in mind the Kyoto Protocol on reducing greenhouse gas emissions into the atmosphere [2], it is a matter of time when the emission of carbon dioxide $\left(\mathrm{CO}_{2}\right)$ from the Diesel engines will be limited.

With the introduction of legislation on limiting emissions of Diesel engine, a various studies have been conducted in order to reduce the emissions. Researches on optimizing the combustion process in the cylinder by improvements of the engine (combustion chamber shape, the flow in the cylinder, the fuel injection mode, etc.) have led to a reduction in the so-called "raw emissions" [3]. Studying the fuel, reducing sulfur content and constant improvement of fuel quality led to reducing emissions also. However, studies have shown that the harmful emission is most reduced by conducting the purification of exhaust gases after they came out of the engine, i.e by subsequent processing.

With the appearance of the Euro 1 the amount of carbon monoxide (CO) and unburned hydrocarbons (HC) is reduced by using an oxidation catalyst that is built into the Diesel engine [4]. Since the Diesel engine is running lean, due to the large excess air in the exhaust gases there was no possibility of supervising the catalyst lambda probe, i.e. was not included in the OBD program, and engine control unit (ECU) had no information on the condition of the exhaust gas. In order to reduce the content of nitrogen oxides (NOx), a system for exhaust gas recirculation into the intake manifold Diesel Engine EGR was investigated and successfully implemented, which lowers the temperature of combustion in the cylinder, and thus the creation of nitrogen oxides (NOx) [5, 6]. When the Euro 5 entered the force in 2009, the permitted amount of particulate matter (PM) emission in the Diesel engine was significantly reduced. Researches in order to solve this problem [7] led to the development of so-called Diesel Particulate Filter (DPF) that, by its shape keeps solids in it, and from time to time is emptied by the combustion of accumulated particles by means of exhaust gases high temperature, resulting from the late fuel injection during the expansion stroke. Euro 6 normative, that entered the force in 2014, significantly reduces and limits the amount of nitrogen oxides (NOx) emission in the Diesel engine. In recent times, in order to reduce NOx emissions, a selective catalytic reduction (SCR) is more and more researched, developed and operated $[8,9]$. The SCR system for reduction of nitrogen oxide uses the liquid reducing agent on the basis of ammonia $\left(\mathrm{NH}_{3}\right)$ which is injected into the exhaust pipe before the reduction catalyst and in the catalyst leads to chemical reactions which transform harmful nitrogen oxides into nitrogen $\left(\mathrm{N}_{2}\right)$ and water $\left(\mathrm{H}_{2} \mathrm{O}\right)$. Concern for the protection of the human environment has become one constant race between the applicable legal regulations and techniques that must follow.

This paper discusses the problem of increased emissions of harmful exhaust gases of the Diesel engine in situations where the engine control unit (ECU) has not memorized a single error that indicates a malfunction in the control system of the engine. This issue was investigated on the Diesel engines whose control units (ECU) are connected, via CAN-Bus connection [10], with the control devices of other systems in the vehicle.

\section{Development of the algorithmic model}

Just absolutely correct operation of the Diesel engine, with the all elements of the control system correctly functional, guarantees the emissions within the prescribed limits. Any disruption in the engine, as well as a malfunction of individual components, causes improper combustion which results in an increase in harmful emissions. If the emission of harmful exhaust gases of the Diesel engine is increased, for reasons of failure of one or more components of the control system, the problem is detected by commonly known diagnostic methods and by removing detected defection the engine gas emission returns to the acceptable range.

However, there are situations when the Diesel engine has increased emissions of harmful exhaust gases and no component of the system of electronic engine management has any visible deficiency and no electrical malfunction in the form of errors stored in the engine control unit, which is read by diagnostic device. The consequence of increased emissions in these circumstances may be the wrong signal of the sensor which is within the limits of the possible values so engine control unit doesn't recognize it as a mistake and a part of the regulatory process in the work of the Diesel engine is performed towards it. Another option, which can also lead to increased emissions, is the imprecise work of the actuator even though they are regularly triggered by the control unit pulse signal; hence error related to their electrical malfunction is not recorded.

In order to detect these hidden problems in the Diesel engine, which directly affect the increase in its emissions, this paper has developed an optimal algorithmic model for system diagnostics of Diesel engines with electronic control exhaust gases after-treatment and its abbreviated version is shown in Figure 1.

Through a developed comprehensive algorithmic model for system diagnostics of Diesel engines with electronic control exhaust gases after-treatment, individual system components of the engine management test are done step by step, which ultimately leads to detection of the problem that directly affects the increase of the soot particles in the exhaust. By the complete algorithm model, among other, testing of differential pressure sensor, pressure sensor in the intake manifold, fuel injectors, clutch pedal position sensor, speed sensor engine, Hall sensor, EGR valve, turbocharger control, throttle the suction pipe, temperature sensors on DPF filter, is planned as well as the testing of all other elements of the engine whose malfunction leads to increased formation of soot particles and an increase in the total emission of harmful exhaust gases. 


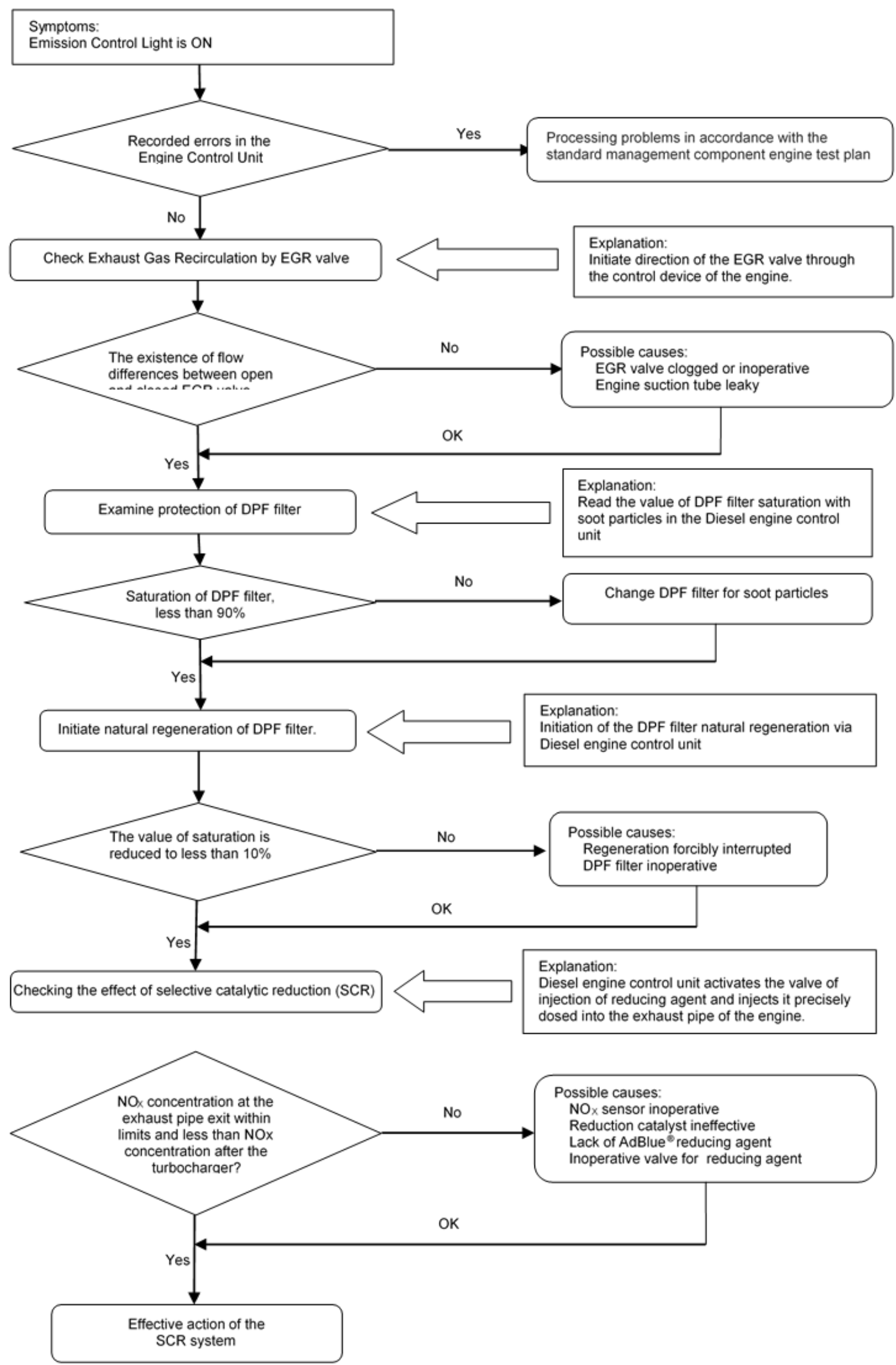

Fig. 1. Algorithm model for exhaust gases system diagnostics 


\section{Experimental setup}

Functionality check of the developed algorithm model was done experimentally on 2.0 TDI and 1.6 TDI Diesel engines, type Volkswagen with electronic control, Common Rail fuel injection system, built-in system of exhaust gas recirculation in the intake manifold of the engine (EGR), oxidation catalyst, Diesel particulate filter (DPF) and selective catalytic reduction (SCR). In the process of measuring, testing and diagnosis during experimental research measurement and diagnostic equipment shown in Figure 2 was used.

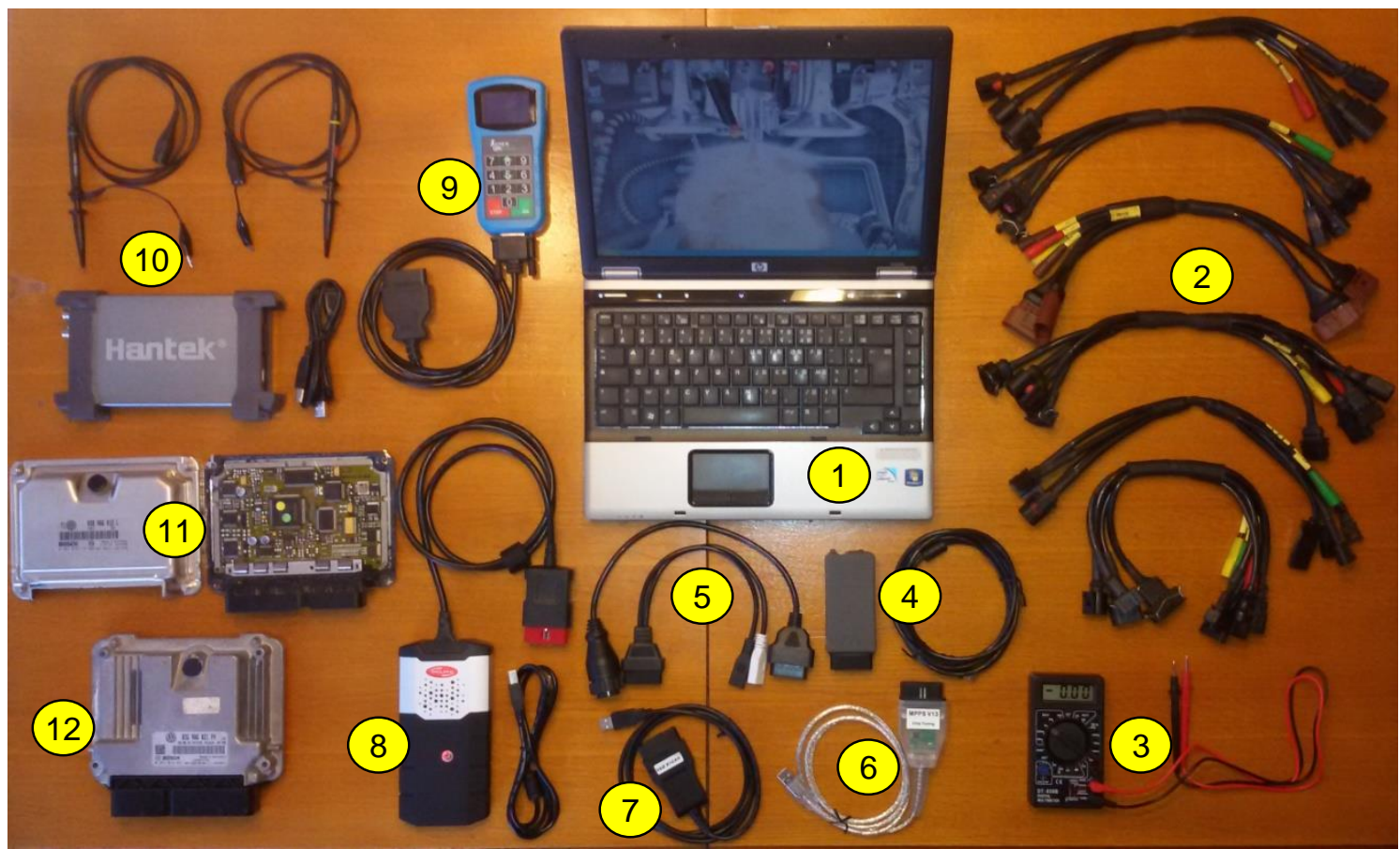

1. Diagnostic device (computer with software)

2. Adapter cables for testing sensors and actuators

3. Multimeter (voltmeter, ammeter and ohmmeter)

4. OBD Bluetooth diagnostic head

5. Adapters for OBD diagnostic connector

6. Remap tool to modify the Engine control unit software (Chip-tuning)
7. OBD diagnostic cable for reading EEPROM of the Engine control unit

8. Diagnostic adapter for reading engine operation

9. OBD $\mathrm{K}+\mathrm{CAN}$ device for reading codes

10. Dual-channel memory oscilloscope

11. EDC controller for Diesel motor

12. EDC controller for Diesel motor

Fig. 2. Measuring and diagnostic equipment used in the experiment

\section{Research results}

Experimental testing of the functionality of the developed algorithmic model for exhaust after-treatment system diagnostics is carried out in accordance with the anticipated test steps within the model. All the components (sensors and actuators) in the Diesel engine operation management system and in the exhaust after-treatment systems were tested. The deviation of the measured actual parameters of the engine in relation to the required parameters indicate malfunction of the Diesel engine, and thus the increased amount of harmful emissions in the exhaust gases.

In this paper, segments of the experimental testing of algorithmic models for exhaust after-treatment system diagnostics are stated as an example. Any disturbance in the regulation of the start and fuel injection quantity leads to incomplete combustion in the cylinder of the Diesel engine which results in an increase in emissions [11]. The amount of fuel injection is directly dependent on the duration of injection through the injectors. In order to test the algorithmic model presented in this paper, experimental testing process of control fuel injection was done. On the tested Diesel engine

was established duration of the main and three subsequent injections during the running regeneration of DPF filter at intervals of about 20 to 30 seconds. Piezo fuel injectors that, with an electric pulse, trigger engine control unit via the piezo actuator were used on the tested engine. It has been found that the duration of the main injection was longer in the beginning of the regeneration process (about $300 \mu \mathrm{s}$ ) while the later has become shorter (about $290 \mu \mathrm{s}$ ). Experimentally determined duration of the main injection is expected, because the beginning of the regeneration process requires a greater amount of fuel to increase the exhaust temperature. Diagram of the main fuel injection during the regeneration is shown in Figure 3. The duration of subsequent injections at time intervals is shown in Figure 4. The diagram shows that the third post-injection had interruptions at certain points (injection duration value is 0 ), which had resulted in a slight disturbance in the exhaust after-treatment. 


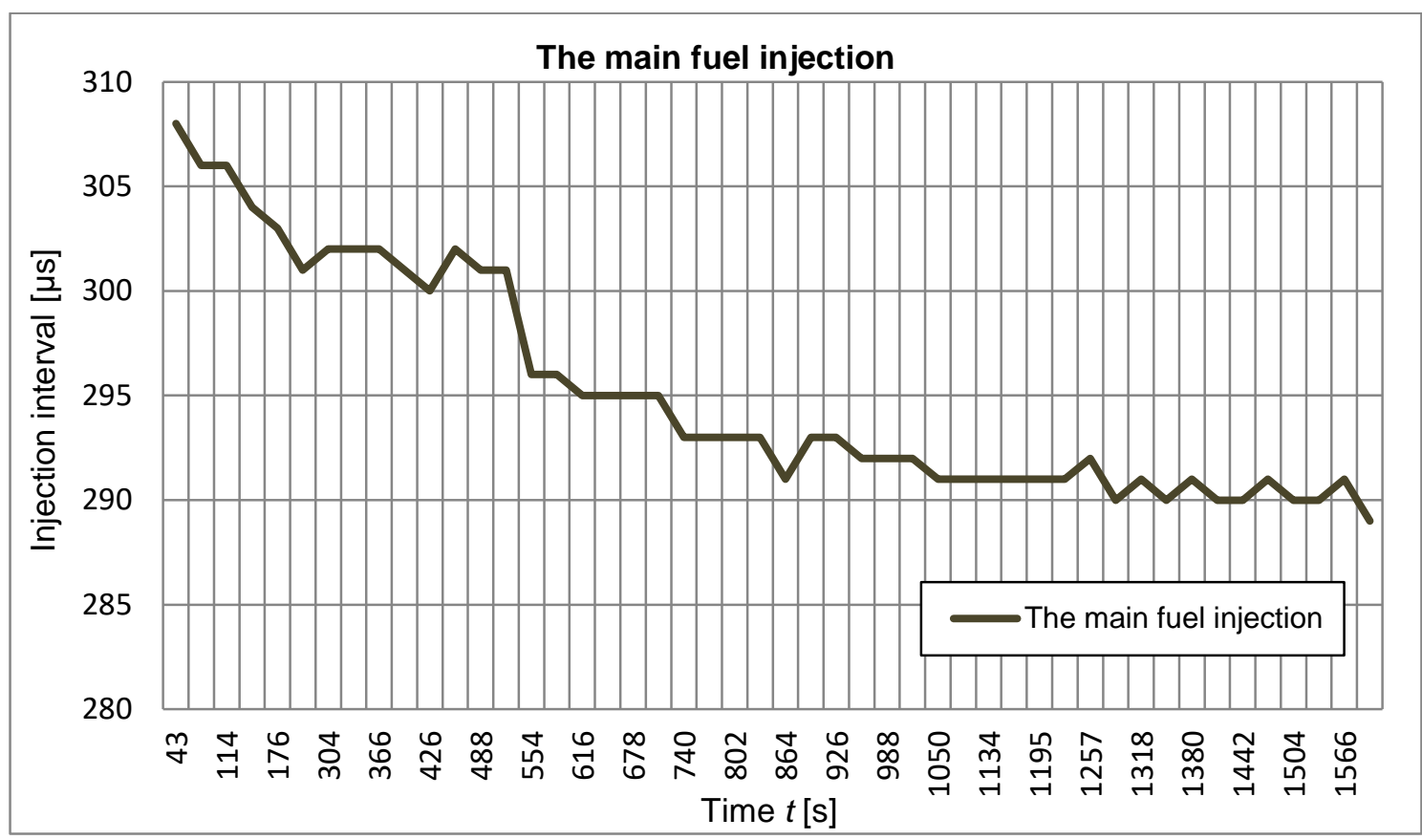

Fig. 3. The main fuel injection during DPF regeneration

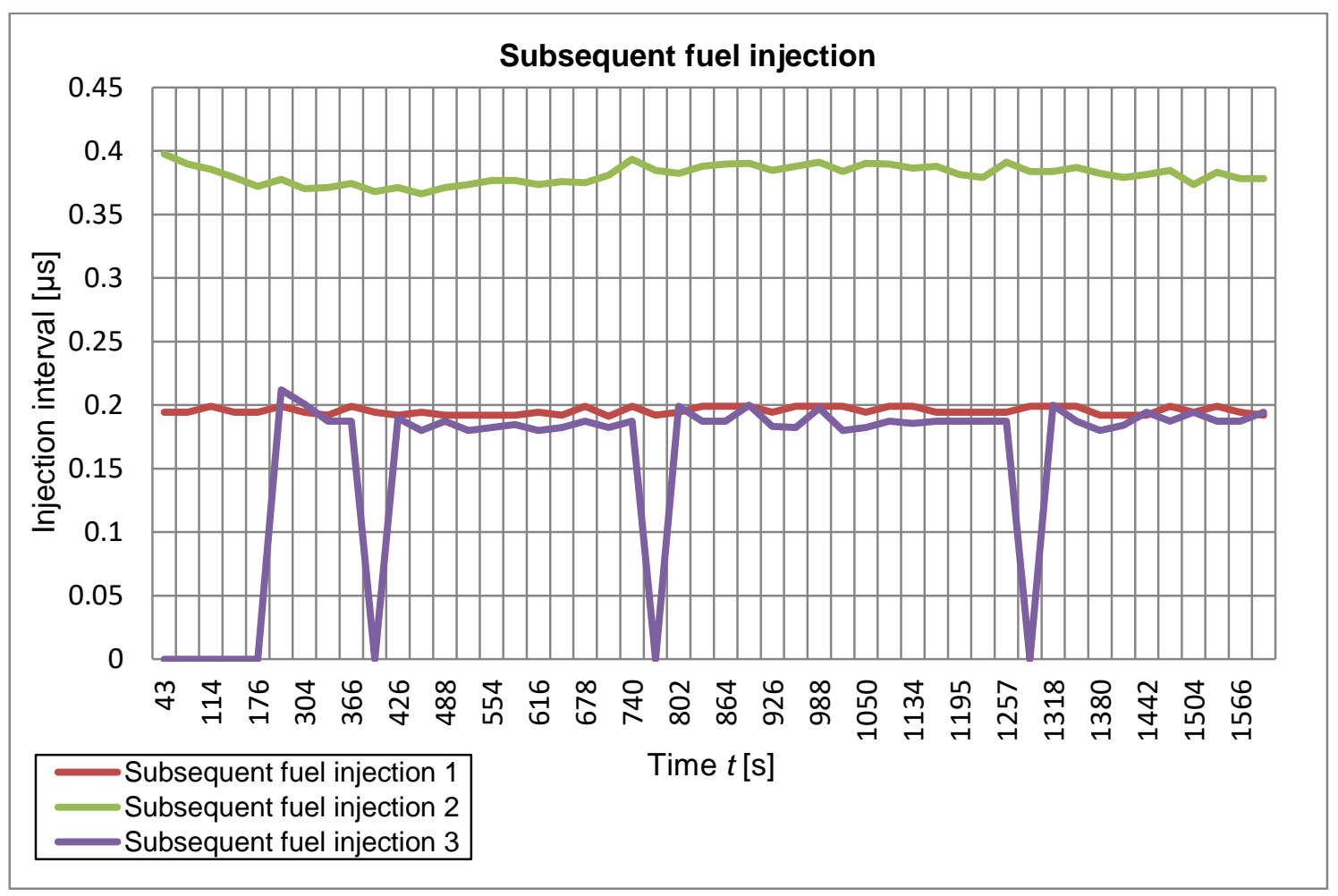

Fig. 4. The subsequent fuel injection during DPF regeneration

In the functionality check of the DPF filter, the element that most affects the reduction of soot particles, PTC temperature sensors in front of the turbocharger, before and after the DPF were tested. From the correctness of the sensor signal, depends the very process of regeneration in the DPF filter. In the process of forced regeneration, the soot particles deposited in the DPF filter burn due to an increase in the exhaust gas temperature conducted by the control device of the engine. In the experiment, during the process of regeneration, at intervals of about 20 seconds, temperature values of the exhaust gases were measured at three characteristic places (in front of the turbocharger, before and after the DPF Filter). The measured temperature values from the beginning of the process of regeneration (43 seconds after the warm-up phase) until the end of the regeneration process (1597 seconds after the start of the process) are shown in Figure 5. The continuous change in the value of temperature indicated the correctness of the tested temperature sensor. Each illogical abrupt change 
in temperature indicates a failure of any of the used temperature sensors which directly affects the process of regeneration and the creation of high emissions of soot.

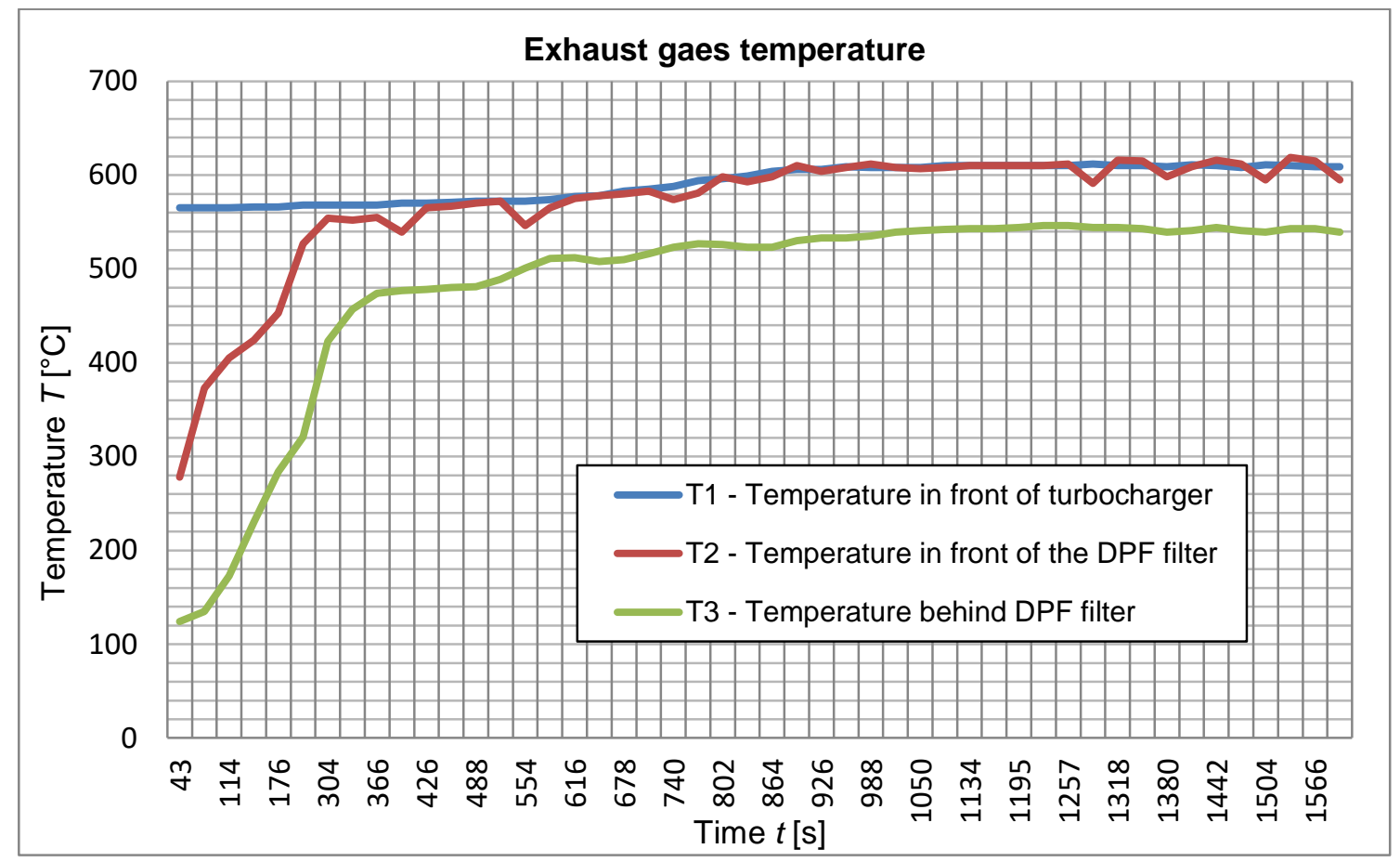

Fig. 5. Exhaust gases temperature change during DPF regeneration

In the process of experimental test of the developed diagnostics model, one of the steps was to monitor changes in the saturation of DPF filter with soot particulate. During the process of regeneration amount of soot particles must be continuously reduced as a result of combustion in the DPF filter. The mass of soot, on a tested Diesel engine, before the beginning of the regeneration was 24 grams, and the concentration is measured by the differential pressure sensor. Values of the mass of soot in the DPF filter, were measured at intervals during the regeneration and recorded in the Table 1.

\begin{tabular}{|l|r|r|r|r|r|r|r|r|}
\hline \multicolumn{1}{|c|}{ Dimension } & \multicolumn{7}{c|}{ Value } \\
\hline Time $t[\mathrm{~s}]$ & 43 & 83 & 114 & 145 & 176 & 204 & 304 & 335 \\
\hline Soot mass [g] & 24,12 & 24,12 & 24,12 & 24,12 & 24,12 & 24,12 & 24,00 & 23,82 \\
\hline Time $t[\mathrm{~s}]$ & 366 & 395 & 426 & 457 & 488 & 516 & 554 & 585 \\
\hline Soot mass [g] & 23,42 & 23,08 & 22,58 & 22,00 & 21,38 & 20,76 & 19,78 & 19,08 \\
\hline Time $t$ [s] & 616 & 647 & 678 & 709 & 740 & 771 & 802 & 833 \\
\hline Soot mass [g] & 18,12 & 17,18 & 16,26 & 15,32 & 14,30 & 13,46 & 12,42 & 11,38 \\
\hline Time $t$ [s] & 864 & 895 & 926 & 957 & 988 & 1019 & 1050 & 1079 \\
\hline Soot mass [g] & 10,44 & 9,42 & 8,36 & 7,50 & 6,70 & 6,00 & 5,44 & 4,96 \\
\hline Time $t[\mathrm{~s}]$ & 1134 & 1165 & 1195 & 1226 & 1257 & 1287 & 1318 & 1349 \\
\hline Soot mass [g] & 4,22 & 3,90 & 3,64 & 3,40 & 3,20 & 3,02 & 2,88 & 2,74 \\
\hline Time $t[\mathrm{~s}]$ & 1380 & 1411 & 1442 & 1473 & 1504 & 1535 & 1566 & 1597 \\
\hline Soot mass [g] & 2,60 & 2,48 & 2,36 & 2,24 & 2,14 & 2,04 & 1,92 & 1,84 \\
\hline
\end{tabular}

Table 1. Measured values of soot mass during DPF regeneration

According to Table 1, each point of observation, in the process of regeneration, is associated with the value of the mass of soot at the time. The table clearly shows that during the experimental testing of the process of regeneration DPF passed without interference because the value of the mass of soot in the filter is continuously decreased without sudden changes. At the end of the regeneration process, combustion of soot particles was complete because the value of soot fell below 2 grams. This indicates the correctness of the DPF filter and overall system exhaust after-treatment. Also, the outcome of the process indicates the correctness of the entire Diesel engine management system. 
The conducted experiments in this paper, in accordance with the steps of developed algorithm model for exhaust gases system of Diesel engine with electronic control diagnostics, has shown that any malfunction of the Diesel engine, that affects the harmful emissions, can be detected by this systematic approach.

\section{Conclusion}

After the research, it has been experimentally demonstrated and supported by diagnostic protocol, that the developed algorithmic model for exhaust gases system of diesel engine with electronic control diagnostics control is fully functional and effective. The results of experimental studies in this paper clearly and unequivocally indicate that using the developed algorithm model for exhaust gases system of Diesel engine with electronic control diagnostics, which includes interactive communication of engine control unit and engine diagnostic device, allows accurate diagnosis of faults and disturbances in the system exhaust after-treatment. Also, the developed model can specify the impact of failure of each individual component of the Diesel engine with electronic control in the process of after-treatment of exhaust gases of the engine. In the future, it is certainly expected emergence of new more sophisticated systems for Diesel engines exhaust gas after treatment, therefore the future goal of the research will be the adjustment of the algorithmic model to those systems.

\section{References}

[1] EUR-Lex: Regulation (EC) No 715/2007 of the European Parliament and of the Council on type approval of motor vehicles with respect to emissions from light passenger and commercial vehicles (Euro 5 and Euro 6) and on access to vehicle repair and maintenance information, Annex I. Emission Limits, Official Journal of the European Union, No. 284, 20.06.2007.

[2] United Nations: Kyoto Protocol to the United Nations Framework Convention on Climate Change, UNFCCC, Kyoto, 1998.

[3] Takeda, Y.; Keiichi, N.: Emission Characteristics of Premixed Lean Diesel Combustion With Extremely Early Staged Fuel Injection, SAE Technical Paper 961163, Dearborn, 1996.

[4] Bagly, S.; Gratz, L.; Johnson, J.; McDonald, J.: Effects of an Oxidation Catalytic Converter and a Biodiesel Fuel on the Chemical, Mutagenic, and Particle Size Characteristics of Emissions from a Diesel Engine, Environmental Science and Technology, Vol. 32, No. 9, pp 1183-1191, 1998.

[5] Dürnholz, M.; Eifler, G.; Endres, H.: Exhaust Gas Recirculation - A Measure to Reduce Exhaust Emissions of DI Diesel Engines, SAE Technical Paper 920725, Detroit, 1992.

[6] Sabau, A.; Barhalescu, M.; Oanta, E. \& Dumitrache, C., Simplified Model for Combustion Reactions in Diesel Engine, Annals of DAAAM for 2009 \& Proceedings of the 20th International DAAAM Symposium, pp. 0811-0812, B. Katalinic (Ed.), Published by DAAAM International Vienna, 2009.

[7] Adler, J.: Ceramic Diesel Particulate Filters, International Journal of Applied Ceramic Technology, Vol. 2, No. 6, pp 429-439, 2005.

[8] Brandenberger, S.; Krocher, O.; Tissler, A.; Althoff, R.: The State of the Art in Selective Catalytic Reduction of $\mathrm{NO}_{\mathrm{x}}$ by Ammonia Using Metal-Exchanged Zeolite Catalysts, Catalysis Reviews: Science and Engineering, Vol.50, No. 2, pp 492-531, 2008.

[9] Kyu Yun, B.; Young Kim, M.: Modeling the selective catalytic reduction of $\mathrm{NO}_{\mathrm{x}}$ by ammonia over a Vanadia-based catalyst from heavy duty diesel exhaust gases, Applied Thermal Engineering, Vol. 50, No. 1, pp 152-158, 2013.

[10] Dosek, R.; Janku, P.; Bliznak, M. \& Varacha, P., Secure High Level Communication Protocol for CAN Bus, Proceedings of the 26th DAAAM International Symposium, pp.1009-1015, B. Katalinic (Ed.), Published by DAAAM International, Vienna, 2016.

[11] Blyankinshtein, I.; Voevodin, E. \& Ruban, A., Techniques of Adjusting Opacity Estimates of the Diesel Exhaust Gases, Proceedings of the 26th DAAAM International Symposium, pp.0883-0891, B. Katalinic (Ed.), Published by DAAAM International, Vienna, 2016. 